\title{
Las herramientas web 2.0 en el desarrollo de habilidades lectoras
}

\author{
Web 2.0 tools in the development of reading skills
}

\author{
Ferramentas da web 2.0 no desenvolvimento de habilidades de leitura
}

\section{ARTíCULO DE INVESTIGACIÓN}

Danny S. Delgado Togra

santiago_23_90@hotmail.es

https://orcid.org/0000-0002-1067-3589
José M. Salazar Moreno

marlon.salazar@educacion.gob.ec

https://orcid.org/0000-0001-5416-8309

Laura d.A. Herrera Herrera

angeles.herrera@educacion.gob.ec

https://orcid.org/0000-0001-5132-3422

Ministerio de Educación de Ecuador, Guayaquil, Ecuador

\section{RESUMEN}

El presente artículo aborda el tema de las habilidades lectoras de los estudiantes de tercero de bachillerato de la Unidad Educativa Fiscal Martha Bucaram de Roldós, ubicada en la parroquia Tarqui al norte de la ciudad de Guayaquil. Las habilidades lectoras se analizaron por medio de un conjunto de indicadores o dimensiones y la influencia con las herramientas Web 2.0, mediante la integración de estas en un entorno virtual elaborado en Moodle para potenciarlas. La investigación tuvo un enfoque cuantitativo - cualitativo, su alcance fue descriptivo - correlacional, el universo elegido para esta investigación fue de 218 estudiantes de las jornadas matutina y vespertina de la Institución. Para el desarrollo de la investigación se utilizó un instrumento validado por 5 expertos obteniendo un IVC 0.99 y un alfa de Cronbach de .732. Una vez analizados los datos se evidenció un alto dominio de habilidades y nivel lector de los participantes teniendo el $43 \%$ un nivel excelente y $56 \%$ adecuado con solo $1 \%$ de la población en deficiente.

Palabras clave: TIC; Habilidades lectoras; herramientas Web 2.0

\section{ABSTRACT}

This article addresses the issue of reading skills of third year high school students of the Martha Bucaram de Roldós Public Education Unit, located in the Tarqui parish in the north of the city of Guayaquil. Reading skills were analyzed by means of a set of indicators or dimensions and the influence with Web 2.0 tools, through the integration of these in a virtual environment developed in Moodle to enhance them. The research had a quantitative-qualitative approach, its scope was descriptive-correlational, and the universe chosen for this research was 218 students of the morning and afternoon sessions of the Institution. For the development of the research an instrument validated by 5 experts was used, obtaining a CVI 0.99 and a Cronbach's alpha of .732. Once the data were analyzed, a high level of reading skills and reading level of the participants was evidenced, with $43 \%$ having an excellent level and $56 \%$ an adequate level, with only $1 \%$ of the population being deficient.

Key words: ICT; Reading skills; Web 2.0 tools

\section{RESUMO}

Este artigo trata da capacidade de leitura dos alunos do terceiro ano do ensino médio na Unidade Pública de Educação Martha Bucaram de Roldós, localizada na paróquia de Tarqui, ao norte da cidade de Guayaquil. As habilidades de leitura foram analisadas por meio de um conjunto de indicadores ou dimensões e a influência das ferramentas Web 2.0, através da integração destas em um ambiente virtual desenvolvido no Moodle para melhorá-las. A pesquisa teve uma abordagem quantitativaqualitativa, seu escopo foi descritivocorrelacional, o universo escolhido para esta pesquisa foi de 218 estudantes das sessões da manhã e da tarde da instituição. Para o desenvolvimento da pesquisa foi utilizado um instrumento validado por 5 especialistas, obtendo um CVI 0,99 e um alfa de Cronbach de 0,732. Uma vez analisados os dados, verificouse que os participantes tinham um alto nível de habilidade de leitura e nível de leitura, com $43 \%$ a um nível excelente e $56 \%$ a um nível adequado, com apenas $1 \%$ da população a um nível deficiente.

Palavras-chave: TIC; Habilidades de leitura; Ferramentas Web 2.0 


\section{INTRODUCCIÓN}

El conocimiento es un concepto más complejo que la información, no se puede reducir a la mera aglomeración de conceptos no relacionados entre sí, es más bien un proceso que implica sentido, organización y estructura (Mansell y Tremblay, 2015). Lograr el desarrollo de individuos funcionales para la sociedad con actitudes críticas y reflexivas no sería posible sin un nivel lector más que adecuado, esté conjunto de destrezas transversales permite la apropiación del conocimiento y dominio de este en varios ámbitos del saber (Arias, Fidalgo y Robledo, 2012). De acuerdo con antecedentes en investigaciones psicolingüísticas como la de Cuetos (1990) han demostrado que la diferencia entre un buen y mal lector radica en el dominio de ciertas tareas lingüísticas como pueden ser la parafasias o el análisis conceptual del texto, mientras que en tareas no lingüísticas son comparables sus habilidades (Vallejo y Flores, 2015).

En el contexto ecuatoriano ha tomado importancia el incremento del capital humano tecnificado que dé respuesta a las constantes demandas de una sociedad digital con miras a la globalización en cuanto a servicios, abastecimiento y por supuesto innovación, siendo este último vital para la competitividad de la industria en ámbitos regionales o mundiales. Es imprescindible que la educación de respuesta a la necesidad producir ciudadanos críticos con alto nivel de competencias laborales y humanas. Como un antecedente el Ecuador participo en el Segundo Estudio Regional Comparativo y Explicativo (SERCE) 2006 de la UNESCO donde se evaluaron competencias de las áreas elementales como fueron: matemáticas, ciencias y lecto escritura, donde en esta última se obtuvo un $23 \%$ dentro del nivel I, el $46 \%$ en el nivel II práctico de lectura, el $25 \%$ en el nivel III y solo $3 \%$ alcanzó el IV, teniendo presente que solo $2.3 \%$ de los estudiantes no llegó a ninguno.
Dentro del ámbito local en la Unidad Educativa Fiscal "Martha Bucaram de Roldós", ubicada en la ciudad de Guayaquil, Parroquia Tarqui, Circuito 09D06C2, Zona 8, se evidencian los siguientes problemas: bajo nivel de habilidades lectoras, bajo rendimiento académico en la materia de Lengua y Literatura. El problema se detecta en las pruebas SER BACHILLER 2017-2018. En el componente Lingüístico donde se evaluaron los dominios de Semántica contextual, Elementos de la lengua, Comprensión de textos escritos se obtuvieron los siguientes resultados: Insuficiente $31.3 \%$, Elemental $52.8 \%$, Satisfactorio 14,4\%, Excelente 1,6\% (INEVAL, 2019).

Por lo cual se busca determinar mejorar el nivel de competencias lectoras a fin de buscó diseñar un Entorno Virtual de Aprendizaje que integre herramientas Web 2.0 para el desarrollo de habilidades lectoras de los estudiantes de Tercero bachillerato de la Unidad Educativa Fiscal "Martha Bucaram de Roldós".

Con relación al contexto previo, la lectura es la habilidad o destreza más importante dentro de la formación del estudiante, pues esta es transversal, se utiliza en cualquier ámbito del conocimiento y su alto nivel de dominio potencia el aprendizaje, el pensamiento crítico y el rendimiento académico. Las competencias lectoras como proceso de descodificación y comprensión literal son significativas durante toda la vida del individuo.

Se determinó medir el nivel de habilidades lectoras de los estudiantes de tercero de bachillerato con el fin de reconocer ¿Qué nivel de habilidades lectoras poseen los estudiantes de tercero de bachillerato y en qué medida el uso de herramientas web 2.0 las puede potenciar?, mediante el uso de instrumentos de investigación cuantitativos y análisis de datos, utilizando técnicas de estadística descriptiva para determinar ¿Cómo influyen las herramientas Web 2.0? desarrolladas en un 
Entorno Virtual de Aprendizaje para el desarrollo de habilidades lectoras?. Es pertinente realizar esta investigación con el fin de conocer el nivel real de habilidades lectoras de la población objeto de estudio y desarrollar un entorno virtual de aprendizaje que incluya herramientas Web 2.0 para potenciarlas.

\section{MÉTODO}

Se realizó un estudio bajo paradigma cuantitativa para el análisis de la información debido a que se describieron las dimensiones del problema de investigación, aplicando procedimientos estandarizados para medir las variables de investigación, su alcance fue descriptivo - correlacional y su diseño de tipo no experimental (Hernández, Fernández y Baptista, 2014).

Se tuvo como población a 218 estudiantes entre 15-19 años de ambos sexos, pertenecientes a la Unidad Educativa Fiscal "Martha Bucaram de Roldós", delajornadavespertina del nivelbachillerato en las figuras profesionales de Contabilidad, Informática y General Unificado de la ciudad de Guayaquil, distrito 09D06 “Tarqui-2 Educación”. Se planteó un instrumento con 19 ítems para medir las siguientes dimensiones: Comprensión lectora (ítem 1-7), fluidez lectora (ítems 8-14), velocidad lectora (ítems 15-19), de la lectora, fue validado por juicio de 5 expertos en Lengua y Literatura, mediante prueba Alfa de Cronbach e IVC (índice de validez del contenido) se obtuvo un valor de 0.730 como a valor del Alfa y 0.99 para el IVC (García, 2003).

La técnica de muestreo fue de tipo no probabilístico, esto por conveniencia para la investigación y debido a que no se utilizó fórmula para muestra estratificada, se aplicó el instrumento al total de la población (Hernández et al., 2014), como técnica de recolección de datos se utilizó el cuestionario tipo escala de Likert con cuatro niveles y adaptado a la virtualidad mediante la plataforma de Google Froms.

\section{RESULTADOS}

Para realizar el análisis estadístico de los valores obtenidos por el instrumento se establecieron tres niveles de dominio de habilidades lectoras los cuales fueron: Insuficiente, Adecuado y Excelente. También se determinó el rango y amplitud tomando en cuenta los valores máximos y mínimos de acuerdo con el reactivo siendo estos 19 puntos como valor mínimo y 76 puntos como máximo (Frías, 2020). Teniendo los siguientes rangos para cada nivel y así como también el criterio de interpretación.

Table 1. Rangos y criterios de interpretación del instrumento.

\begin{tabular}{clc}
\hline Nivel & \multicolumn{1}{c}{ Interpretación } & Puntaje \\
\hline Excelente & $\begin{array}{l}\text { Utilizar las habilidades lectoras en la comprensión textos completos y las relaciona con otras } \\
\text { áreas del conocimiento. }\end{array}$ & $59-76$ \\
Adecuado & $\begin{array}{l}\text { Utiliza de manera adecuada las habilidades lectoras y puede aplicarlas como herramientas de } \\
\text { comprensión de textos largos y relacionarlas con otras áreas del conocimiento. }\end{array}$ \\
& $\begin{array}{l}\text { No domina en un nivel adecuado de habilidades lectoras o no las puede aplicar de manera } \\
\text { correcta como herramientas de comprensión de textos y no puede relacionarlas con otras } \\
\text { áreas del conocimiento. }\end{array}$ \\
\hline
\end{tabular}


De acuerdo lo expuesto por la Tabla 1, se establecen criterios de interpretación que definen los valores obtenidos por los individuos encuestados, buscado determinar el nivel de dominio en habilidades lectoras promediado de la población. También se determinó el nivel de acuerdo con cada dimensión propuesta dentro del instrumento.
Para la dimensión comprensión lectora que tuvo como ítems de evaluación las preguntas 1 a la 7 que buscaron contrastar el nivel de comprensión lectora dominante de los participantes mediante cuestionamientos objetivos que indagaron en sus hábitos y estilos de lectura, teniendo los siguientes resultados descritos en el Gráfico 1.

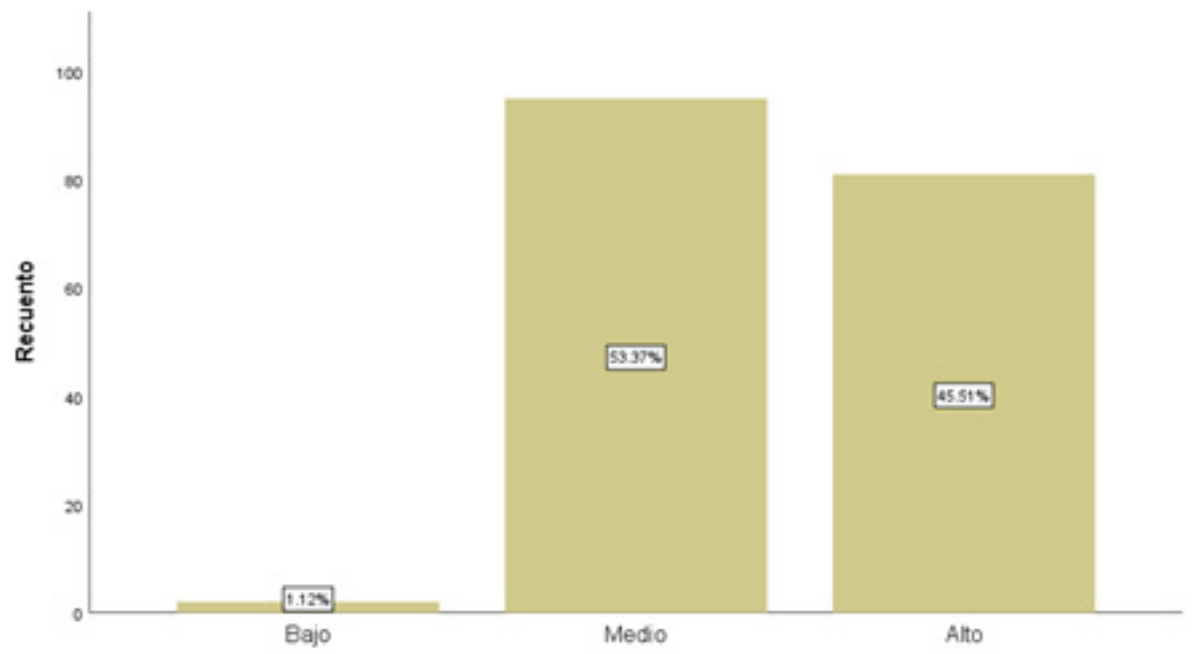

Graph 1. Dimensión comprensión lectora.

En cuanto a la dimensión de la fluidez lectora descrita en el Gráfico 2, se determinó que 59.55\% de los participantes tuvo un nivel adecuado en cuanto a esta habilidad y el 37.08\% alcanzo el nivel máximo de excelente y solo el 3.37\% fue deficiente, este dato permite establecer que la población tiene ciertos inconvenientes en cuanto a la fluidez de la lectura, característica importante para lograr un nivel inferencial de lectura y también permite establecer la necesidad de estimular esta habilidad mediante herramientas digitales.

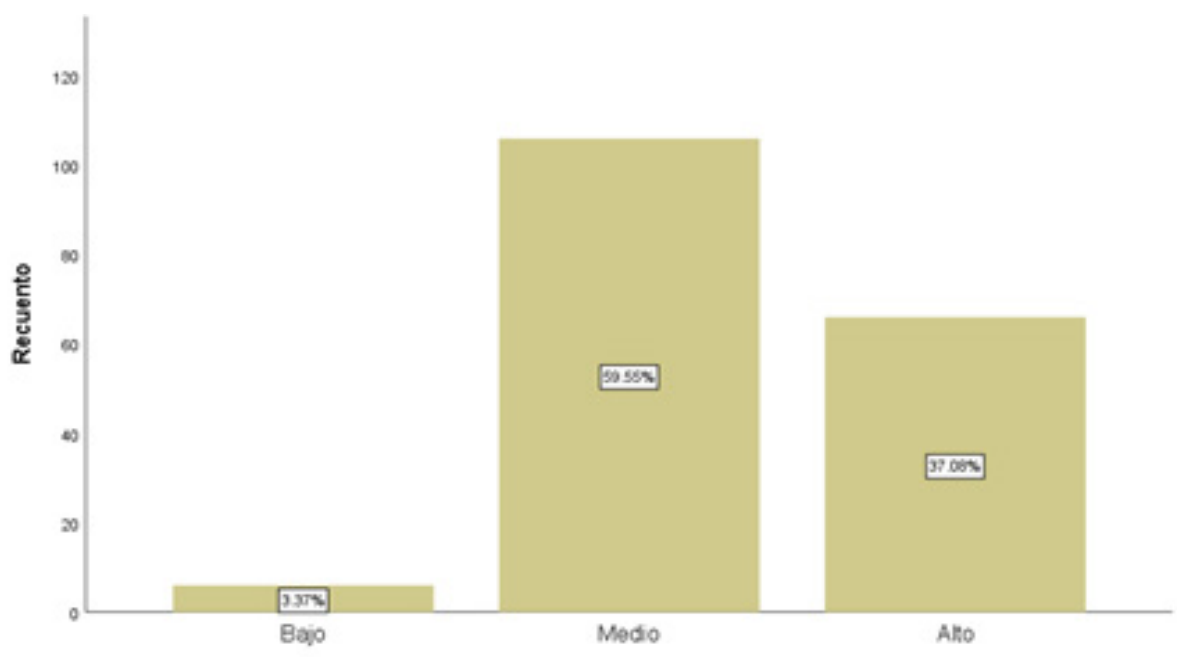

Graph 2. Dimensión fluidez lectora. 
En cuanto a la dimensión de la fluidez lectora descrita en el Gráfico 2, se determinó que 59.55\% de los participantes tuvo un nivel adecuado en cuanto a esta habilidad y el 37.08\% alcanzo el nivel máximo de excelente y solo el $3.37 \%$ fue deficiente, este dato permite establecer que la población tiene ciertos inconvenientes en cuanto a la fluidez de la lectura, característica importante para lograr un nivel inferencial de lectura y también permite establecer la necesidad de estimular esta habilidad mediante herramientas digitales.

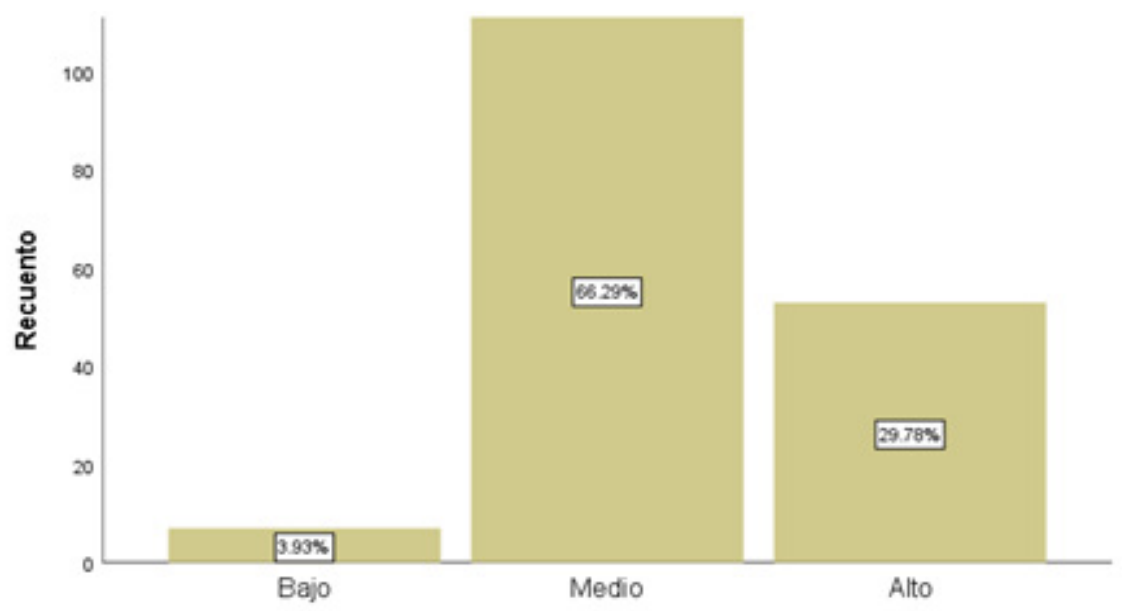

Graph 3. Dimensión velocidad lectora.

En cuanto a la dimensión de velocidad lectora descrita en el gráfico 3 es posible establecer que de acuerdo con el $66.29 \%$ de participantes estos tienen un nivel adecuado en su velocidad lectora y solo $29.78 \%$ de estos logran tener una velocidad excelente de lectura, el $3.93 \%$ de los estudiantes participes del estudio no logra tener un nivel aceptable de velocidad de lectura de textos.
Analizados los niveles de acuerdo con cada dimensión descrita en el instrumento, fue meritorio determinar el nivel general obtenido por los partícipes del estudio el cual se describe en el gráfico 4.

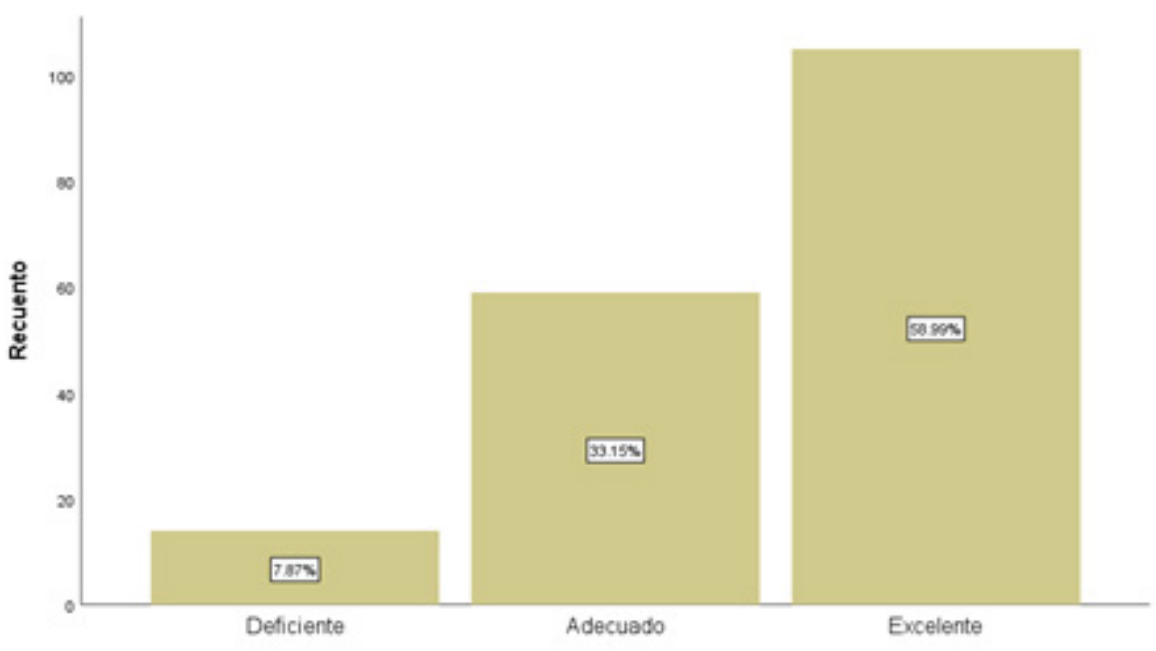

Graph 4. Nivel general de habilidades lectoras. 
De acuerdo con la Gráfica 4 sobre el nivel general de los estudiantes fue excelente en cuanto al 58.99\% del total de participantes, pero el $33.15 \%$ obtuvieron adecuado y el $7.87 \%$ fue deficiente, demostrando que de acuerdo con el contexto del estudio es meritorio aplicar estrategias que integren tecnologías como las de la web 2.0 en el desarrollo de habilidades lectoras de estos dos segmentos de la población (Gutiérrez, 2018).

También se determinó, si existía alguna correlación entre la comprensión y fluidez lectora, para este análisis se midió la normalidad de los valores obtenidos, mediante prueba de estadística inferencial tomando en referencia la significancia siendo esta la probabilidad de que hayan surgido datos sin que haya diferentes entre grupo de valores (Gómez y Roussos, 2012), esta debe estar por debajo de $0.50 \%$, lo cual permite considerar que las pruebas son reales y estadísticamente significantes. Correpondientemente se analizó la naturaleza de los puntajes obtenidos por el instrumento dando como resultado una distribución de valores por tendencia de curva central esto mediante la prueba de Kolmogorov-Smirnov (Cauas, 2015).

Una vez obtenida la distribución de valores se determinó utilizar la prueba del coeficiente $\mathrm{p}$ de Pearson para determinar las correlaciones entre dimensiones debido a su distribución normal.

Table 2. Resultados de la prueba P de Pearson entre comprensión y fluidez lectora.

\begin{tabular}{llcr}
\hline & & Comprensión Lectora & Fluidez Lectora \\
\hline \multirow{2}{*}{ Comprensión Lectora } & Correlación de Pearson & 1 & $.648^{\star *}$ \\
& Sig. (bilateral) & & .000 \\
& $\mathrm{~N}$ & 217 & 217 \\
Fluidez Lectora & Correlación de Pearson & $.648^{* *}$ & 1 \\
& Sig. (bilateral) & .000 & 217 \\
\hline
\end{tabular}

De acuerdo con los resultados de la prueba de correlación de Pearson la relación es positiva fuerte con .648 siendo esta mayor 0.50 , entonces es posible afirmar que en el contexto de investigación existe una fuerte relación entre la fluidez y la comprensión lectora como habilidad, que al dominarse adecuadamente mejora la lectura fluida de textos.

Obtenidos los resultados del análisis de habilidades lectoras de la población objetivo del estudio y validando de acuerdo con estos la necesidad de implementar una propuesta tecnológica instruccional basada en el modelo ADDIE y desarrolla en moodlecloud que mediante herramienta software educativa libre y módulos temáticos orientados al desarrollo de habilidades lectoras mejorarlas (Castillo y Cabrerizo, 2010).

A continuación se muestra el contenido temático o currículo que desarrolló las habilidades adecuadas y deficientes encontradas en los estudiantes el cual fue desarrollado en Tres bloques o unidades las cuales se describen la Tabla 3. 
Table 3. Unidades temáticas del moodle.

\begin{tabular}{|c|c|c|c|c|}
\hline $\mathbf{N}^{\circ}$ & Dimensión & Actividades & Indicador de Logro & Técnica de Evaluación \\
\hline 1 & $\begin{array}{l}\text { Comprensión } \\
\text { Lectora }\end{array}$ & $\begin{array}{l}\text { - Construcción de oraciones y cohesión. } \\
\text { - Razonamiento y conocimiento previo. } \\
\text { - La memoria funcional y la atención. } \\
\text { - Vocabulario. }\end{array}$ & $\begin{array}{l}\text { Comprende el contexto } \\
\text { de fragmentos de textos } \\
\text { mediante la relación } \\
\text { de lecturas previas y } \\
\text { vocabulario. }\end{array}$ & $\begin{array}{l}\text { Foro de opinión } \\
\text { Actividad autónoma } \\
\text { Examen }\end{array}$ \\
\hline 2 & Fluidez Lectora & $\begin{array}{l}\text { - Reconocimiento fonológico de términos } \\
\text { textuales. } \\
\text { - Vocabulario } \\
\text { - Comprensión literal de textos narrativos. }\end{array}$ & & $\begin{array}{l}\text { Foro de opinión } \\
\text { Actividad autónoma } \\
\text { Examen }\end{array}$ \\
\hline 3 & Velocidad Lectora & $\begin{array}{l}\text { - Lectura rápida de textos contra reloj. } \\
\text { - Preguntas dirigidas en razón a lectura. }\end{array}$ & $\begin{array}{l}\text { Desarrolla e integra } \\
\text { contenidos digitales } \\
\text { en experiencias de } \\
\text { aprendizaje. }\end{array}$ & $\begin{array}{l}\text { Foro de opinión } \\
\text { Actividad autónoma } \\
\text { Examen }\end{array}$ \\
\hline
\end{tabular}

Fuente. Elaborado a partir de Elosúa, García, Gómez, López, Pérez y Isabela (2012).

Desarrollados las unidades descritas en la tabla anterior durante treinta días continuos y monitoreados de forma sincrónica se procedió a realizar una evaluación en base a actividades y proyecto integrador que midió las dimensiones descritas anteriormente se pudo determinar que los participantes mejoraron de forma significativa sus habilidades lectoras y también que utilizar herramientas Web 2.0 mejora significativamente el aprendizaje y para el caso del desarrollo de habilidades lectoras demuestra excelentes resultados en ámbitos como la motivación y el desempeño escolar (Mendoza y Molano, 2015).

\section{Discusión}

El desarrollo de una adecuada comprensión lectora mejora de forma significativa las oportunidades de lograr un mayor grado de educación formal, en cuanto al contexto analizado en la presente investigación, las pruebas Ser bachiller régimen costa del periodo académico 2017-2018 en cuanto al dominio lingüístico se obtuvo que el $44.0 \%$ de los estudiantes escolarizados obtuvieron un puntaje promediado entre 7 - 7.99 ubicándolos en el criterio de Elemental y $21.9 \%$ obtuvo un puntaje de 0 a 6.99 colocándolos en Insuficiente, teniendo solo $4.8 \%$ de los participantes obtuvieron un promedio 9.50 a 10 o Excelente (INEVAL, 2019). Estas cifras no se alejan del estudio SERCE 2006 donde también la población escolar se ubicó por debajo de la media de Latinoamérica en el dominio lingüístico junto con países como Guatemala, Hondura, Nicaragua y Panamá, esto en la comparativa del 6to grado de Educación General Básica o EGB y solo pudo aparecer en la media latinoamericana el 3ero de EGB.

En particular al contexto de la investigación, en la UEF "Martha Bucaram de Roldós" los datos publicados por INEVAL (2019) en cuanto al proceso de Ser Bachiller del periodo antes mencionados demostraron que el $51.3 \%$ de estudiantes obtuvieron una nota comparable al criterio de Elemental y el $29.2 \%$ al de Insuficiente, tan solo $0.8 \%$ de los estudiantes postulantes de ese proceso alcanzo el criterio de Excelente. 
En cuanto a los resultados obtenidos por esta investigación se obtuvo que $33.15 \%$ y el $7.87 \%$ de estudiantes de tercero de bachillerato del periodo 2020-2021 están en nivel Adecuado e Insuficiente, siendo esta muestra comparable con los estudiantes participes de la prueba Ser Bachiller del periodo 2017-2018, teniendo en consideración que solo se investigó el nivel de habilidades lectoras y el nivel lingüístico de la prueba comparada tiene una grado mayor de dificultad es notorio que el nivel lector no está bien desarrollado en estos estudiantes.

También el nivel de oralidad del lenguaje en estos estudiantes es bajo pues en las pruebas de fluidez mostraron una mayor dificultad en resolverlas y como la prueba correlacional demostró que en la población de estudio tiene una alta correlación del nivel lector con la fluidez es posible argumentar que presentan problemas de fonética y sintáctica en impiden que lleguen a niveles superiores de comprensión lectora.

Estimular los procesos lectores utilizando herramientas tecnológicas es fundamental en particular de acuerdo con las condiciones actuales provocadas por la digitalización de la sociedad, la lectura clásica o física está desapareciendo, atraer al estudiante hacia el proceso lector es clave en la formación de ciudadanos críticos y proponentes de cambios para la sociedad, el desarrollar habilidades de lectura en medios digitales debe ser un tema de interés para el maestro actual (Gutiérrez y Montes, 2015).

\section{CONCLUSIONES}

De acuerdo con las interrogantes propuestas para la presente investigación y en concordancia con el objetivo planteado, los cuales buscaron determinar el nivel de habilidades lectoras y si el uso de herramientas web 2.0 podía potenciarlas. El nivel diagnosticado por el instrumento de medición y de acuerdo con la escala propuesta, determinó que los estudiantes o la población del estudio tienen un nivel Adecuado en el uso o dominio de habilidades como: Fluidez, Velocidad y Comprensión Lector.

En cuanto al desarrollo de habilidades lectoras mediante el uso de herramientas digitales como las de la web 2.0 es posible afirmar que en ámbitos de motivación y rendimiento escolar son evidentes sus resultados pues el total de los estudiantes terminó el Moodle propuesto para potenciar las habilidades natas y las no dominadas. También los comentarios acerca del diseño de actividades fueron positivo al ser desarrolladas estas a partir de un modelo de diseño instruccional se limitó la posibilidad de errores de continuidad y se aseguró la retroalimentación efectiva.

La comprensión lectora y sus niveles son elementales para lograr un aprendizaje significativo y funcional para la vida, el aprender es una característica propia de los seres humanos, es un bien inmaterial, de acuerdo a el nivel general obtenido por el instrumento de recolección de datos, los estudiantes poseen habilidades lectores adecuadas a su rango etario, pero al no contar una prueba estandarizada con la cual hacer inferencia entre resultados no es posible determinar si población tiene mejores o mayores habilidades lingüísticas que la comparada del periodo 2017-2018.

\section{REFERENCIAS}

Arias, O., Fidalgo, R., y Robledo, P. (2012). Metacognición y comprensión lectora: conocimiento y uso de estrategias. International Journal of Developmental and Educational Psychology, 2(1), 195-201. Recuperado el 4 de febrero de 2021, de https://bit.ly/3oP1SLr

Castillo, S., y Cabrerizo, J. (2010). Evaluación educativa de aprendizajes y competencias. Madrid, España: PEARSON EDUCACIÓN, S.A. Recuperado el 29 de nov. de 2020, de https://bit. ly/35PANl7

Cauas, D. (2015). Definición de las variables, enfoque y tipo de investigación. En D. Cauas, Definición de las variables, enfoque y tipo de 
investigación (págs. 1-11). Bogotá: Biblioteca electrónica de la universidad Nacional de Colombia, Recuperado el 16 de febrero de 2021, de https://bit.ly/37lZN3I

Elosúa, M., García, J., Gómez, M., López, C., Pérez, E., y Isabela, O. (2012). Habilidades lectoras y rendimiento académico. Fundación Infancia y Aprendizaje, 33, 2, 208. Recuperado el 29 de nov. de 2020, de https://bit.ly/3rBd6pG

Frías, D. (2020). Apuntes de consistencia interna de las puntuaciones de un instrumento de medida. Apuntes de consistencia interna de las puntuaciones de un instrumento de medida. Valencia, España: Universidad de Valencia. Recuperado el 18 de febrero de 2021, de https:// bit.ly/3qAmngs

Galindo, M. (2015). Lectura crítica Hipertextual en la web 2.0. Actualidades Investigativas en Educación, 15(1), 1-29. doi:DOI: dx.doi. org/10.15517/aie.v15i1.16972

García, T. (2003). El cuestionario como instrumento de investigación / evaluación. En T. García, Etapas del Proceso Investigador: INSTRUMENTACIÓN. (págs. 1-28). Badajoz, España: Almendralejo, Recuperado el 18 de enero de 2021, de https://bit.ly/3qw31sI

Gómez, J. y Roussos, A. (2012). ¿Cómo Sabemos si Nuestros Pacientes Mejoran? Criterios Para la Significancia Clínica en Psicoterapia: Un debate que se renueva. Revista Argentina de Clínica Psicológica, 21, 173-190. Recuperado el 2021 de febrero de 2021, de https://bit.ly/3q9ErNq

Gutiérrez, A. y Montes, R. (2015). La importancia de la lectura y su problemática. Revista Iberoamericana de Educación (69), 1-13. Recuperado el 27 de febrero de 2021, de https:// bit.ly/3q1WfKy

Gutierrez, B. y Salmerón, P. (2012). Estrategias de comprensión lectora: enseñanza y evaluación en educación primaria. Comprehension learning strategies: instruction and assessment in elementaryschool,16,1,183-202.España:Revista de Currículum y Formación de Profesorado. Recuperado el 29 de nov., de 2020, de https:// www.redalyc.org/pdf/567/56724377011.pdf

Gutiérrez, C. (2018). Fortalecimiento de las competencias de interpretación y solución de problemas mediante un entorno virtual de aprendizaje. Investigación, desarrollo y innovación, 8(2), 279-293. doi: 10.19053/20278306.v8.n2.2018.7170

Hernández, R., Fernández, C. y Baptista, M. (2014). Metodología dela Investigación Científica (Sexta ed.). México DF, México, México: McGRAWHILL / INTERAMERICANA EDITORES, S.A. DE C.V. Recuperado el 16 de 10 de 2020, de https://bit.ly/37kBmVj

INEVAL. (2019). Resultados Ser Bachiller 20172018. Instituto Nacional de Evaluación del Ecuador. Quito: INEVAL. Recuperado el 19 de 06 de 2020, de https://bit.ly/3eu47zD

Mansell, R. y Tremblay, G. (2015). La Renovación de la visión de las sociedades del conocimiento para la paz y el desarrollo sostenible (Primera ed.). Sao Paulo: UNESCO. Recuperado el 18 de 10 de 2020, de https://bit.ly/2HjQozj

Mendoza, R. y Molano, L. (2015). Importancia de formar lectores críticos en educación superior. Revista de Docencia e Investigación Espiral, 5(1), 101-116. Recuperado el 4 de febrero de 2021, de https://doi.org/ftb4

Vallejo, P., y Flores, M. (2015). Intervención psicoeducativa en un caso de altas capacidades. Revista de Psicología Clínica Con Niños y Adolescentes, 2(1),69-74. https://bit.ly/3f2oAxx 